\title{
Bowel obstruction secondary to mesenteric cyst formation
}

\author{
ALBERT H. OLIVENCIA-YURVATI, DO \\ STEVEN H. LEIFHEIT, DO \\ DONALD M. PETERSON, DO
}

\begin{abstract}
A rare cause of intraabdominal obstruction, mesenteric cysts derive from lymphatic tissue. The differential diagnosis should include mesenteric cyst when the patient presents with a history of multiple episodes of partial small-bowel obstruction or with an asymptomatic abdominal mass. A year-old girl was brought to the Family Practice Center with episodic, sharp, nonradiating, left-sided, mid-upper-abdominal pain. Examination indicated a possible diagnosis of mesenteric adenitis. Due to variation in the signs and symptoms of the condition, it appears that the use of ultrasonography is the most effective, nonoperative method of evaluation. Ultrasonography appears to be the most effective, nonoperative method of evaluation. Surgery is the only definitive diagnostic and therapeutic modality.
\end{abstract}

There are many theories concerning the formation of mesenteric cysts, which are derivations of lymphatic tissue that arise in the mesentery, mesocolon, and omentum. Most investigators attribute the cause to be congenitally malformed or malpositioned lymphatic tissue, while others blame failure on the leaves of the mesentery to properly fuse. ${ }^{1}$ Other proposed causes include embryonic abdominal trauma, inflammatory obstruction of existing channels, and localized degeneration of lymph nodes. ${ }^{1}$

The rarity of this condition makes preoperative diagnosis very difficult. ${ }^{2,3}$ In 1986 , Kurtz and associates ${ }^{4}$ found 152 cases of mesenteric or retroperitoneal cyst reported in the English literature between 1950 and 1985; they presented an additional 10 cases, for a total of 162 cases.

With so few cases to draw from, a general practitioner might well omit the possibility of mesenteric cyst in diagnosing a patient with intermittent abdominal pain. Although surgery may present the only obvious therapeutic modality, a definitive diagnosis may be achieved with the use of ultrasonography.

\section{Report of case}

A 4-year-old girl was brought to the Texas College of Osteopathic Medicine Southside Family Practice Clinic because of left-sided, mid-upper-quadrant abdominal pain, which had begun four days earlier. The pain was intermittent, sharp, and nonradiating. Each episode initially had lasted two to three minutes, but the duration increased to 10 minutes. There were pain-free intervals lasting many hours.

The history was negative for trauma, previous surgery, or recent travel. There had been no nausea, vomiting, diarrhea, or constipation. The child's last bowel movement, which had been hard in consistency, had occurred two days earlier. Her appetite was decreased, but she was not anorexic. There were no other related systemic complaints.

The patient was afebrile, and her pulse and respiratory rates were stable. She pointed to the midabdomen as the site of her discomfort. Bowel sounds auscultated in all four quadrants were physiologic in nature. On palpation, a well-defined, movable mass approximately 5 $\mathrm{cm}$ to $6 \mathrm{~cm}$ in diameter was discovered in the left upper quadrant. There was no rigidity, rebound phenomenon, or peritoneal signs; the child complained of slight tenderness on deep palpation. The rectal examination revealed no occult blood or other abnormal findings.

Because of limited laboratory facilities at the clinic, only urinalysis was performed, with the following results: $\mathrm{pH}$ value, 5.0; urobilinogen level, 0.1 Ehrlich unit; +1 proteinuria; and $4+$ ketonuria. The microscopic findings were within normal limits.

The differential diagnosis was narrowed to Meckel's diverticulum, partial small-bowel obstruction, or mesenteric adenitis, and the child was referred to the emergency room at a local hospital.

However, against the physician's advice, the parents took the child home. Some eight hours later, she awoke with severe, unremitting abdominal pain, without nausea or vomiting. She was taken to another local emergency room, where she was found to have an oral temperature of $101^{\circ} \mathrm{F}$ and tachycardia.

On physical examination, the left-sided upper quadrant mass was palpated and peritoneal signs were present. The WBC count was $13,600 / \mathrm{cu} \mathrm{mm}$, with a left shift. Serum electrolyte values were within normal limits, and the serum amylase level also was normal. Plain abdominal films revealed fluid-filled loops of bowel and a mass appropriately $5 \mathrm{~cm}$ to $6 \mathrm{~cm}$ in diameter (Figs 1-4).

The child underwent surgery, which revealed a 6.5$\mathrm{cm}$, globular, cyst-like mass involving the midileum. The 
lesion had caused a volvulus of the small bowel, and approximately 24 inches of markedly ischemic bowel was noted. The small bowel was resected and end-to-end anastomosis was performed. The patient tolerated the procedure well and was discharged on the fifth postoperative day.

Pathologic examination disclosed marked ischemia and mucosal necrosis of the entire segment of resected bowel, and a $6.5-\mathrm{cm}$, lymphogenous mesenteric cyst.

\section{Discussion}

The present case reflects the variation in signs and symptoms of mesenteric cysts. Obviously, the clinical presentation will vary with cyst size and location, as well as with other data related to obstruction. The form and position of the tumor constantly change; therefore, identification is very difficult. In one review, ${ }^{5}$ it was noted that a mass was palpated in $58 \%$ of cases. Many cases have been confused preoperatively with acute appendicitis or appendiceal abscess when the cyst was palpated in the right lower quadrant. ${ }^{2}$

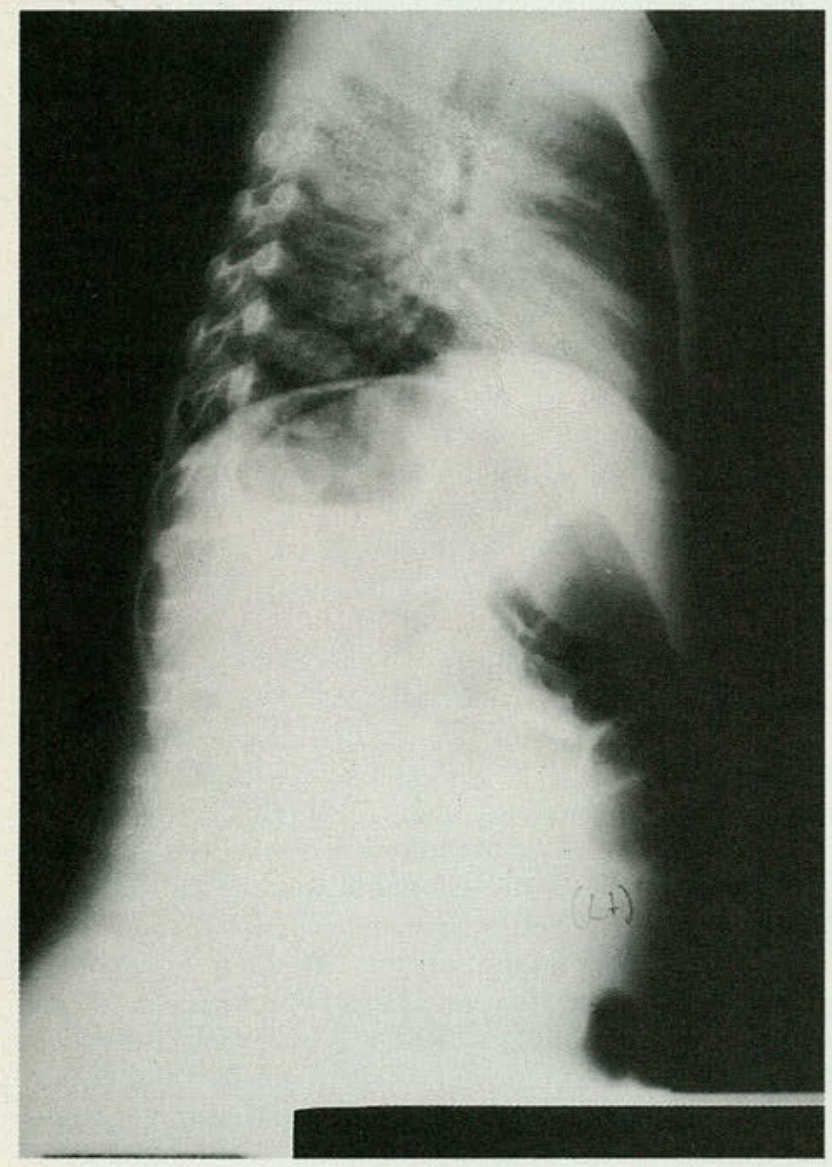

Fig 1. Lateral abdominal chest $x$-ray showing gas and fluidfilled loops of bowel.
Large cysts may cause obvious abdominal distention or a palpable mass, whereas small ones may be completely asymptomatic. The onset of abdominal pain reflects traction in the mesentery, with or without compression or torsion of the small bowel.

The most common presentation is a slowly enlarging, painless abdominal lesion, which causes symptoms only when bowel obstruction occurs. Warfield ${ }^{6}$ states that an abdominal tumor that is rounded, smooth, nontender, and very mobile in the transverse direction is somewhat indicative of a mesenteric cyst.

There are no definitive diagnostic tests. Roentgenography of the abdomen may reveal soft-tissue density or, rarely, calcification. Ultrasonography and computed tomography definitely demonstrates the cystic structure. ${ }^{7}$ And, also, the thin, intracystic septa may be visualized. ${ }^{5}$ Laboratory data that reveals dehydration or malabsorption may lead to suspicion of obstruction from a mesenteric cyst.

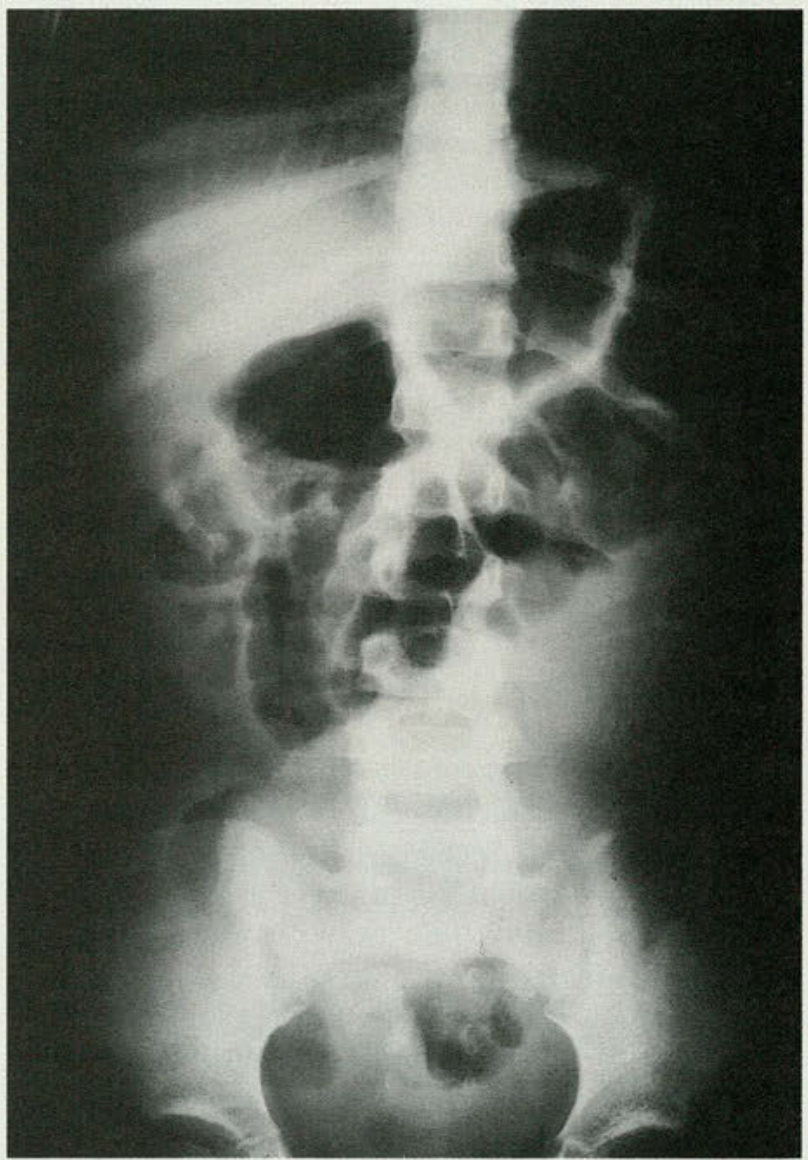

Fig 2. Anteroposterior view of the abdomen showing gas and fluid-filled loops of bowel. 


\section{WHEN CONSIDERING LIFESTYLE-}

LIMITING EFFECTS OF ANTIHYPERTENSIVE THERAPY...

\section{CAN BETA BLOCKER THERAPY LET YOUR PATIENTS "LIVE AS YOUNG AS THEY FEEL"?}




\section{LIVING...AS YOUNG AS THEY FEEL}

\section{COMPARED TO SELECTED BETA BLOCKERS}

ACE inhibitors such as PRINIVIL ${ }^{\circledR}$ (Lisinopril, MSD) are not characteristically associated with:

decreased exercise capacity impotence.*

reduced cardiac output and heart rate malaise, drowsiness

alterations in lipid profile

\section{COMPARED TO TENORMIN}

\section{A recent clinical study' comparing PRINIVIL} and Tenormin demonstrated:

- PRINIVIL 20 to $80 \mathrm{mg}$ produced a much greater reduction in sitting systolic blood pressure ${ }^{\dagger}$ than did Tenormin 50 to $200 \mathrm{mg}(\mathrm{p}<0.01)$, regardless of race.

- PRINIVIL appeared to attenuate diuretic-induced hypokalemia, hyperglycemia, and hyperuricemia.

Significantly fewer patients receiving PRINIVIL required concomitant thiazide therapy $(p<0.05) .^{\dagger+}$

- Significantly fewer patients receiving PRINIVIL had an increase in serum triglycerides $(p<0.05)$.

1. Bolzano, K. et al.: The antihypertensive effect of lisinopril compared to atenolol in patients with mild to moderate hypertension, J. Cardiovasc. Pharmacol. 9(Suppl. 3):S43-S47, 1987.

*Not every product within this therapeutic class has the side effects described.

**Incidence in clinical trials with PRINIVIL was only $0.7 \%$.

Comparable reduction in sitting diastolic blood pressure was seen with PRINIVIL and Tenormin

"The addition of hydrochlorothiazide caused further reductions $(p<0.01)$ in SSBP/SDBP: PRINIVIL, 16.3/12.5 mm Hg; Tenormin, $18.2 / 9.8 \mathrm{~mm} \mathrm{Hg}$

NOTE: Tenormin is a registered trademark of ICI Americas Inc.

PRINIVIL is contraindicated in patients who are hypersensitive to this product and in patients with a history of angioedema related to previous treatment with an ACE inhibitor. All patients should be cautioned that excessive perspiration and dehydration may lead to an excessive fall in blood pressure because of reduction in fluid volume. Other causes of volume depletion, such as vomiting or diarrhea, may also lead to a fall in blood pressure. 


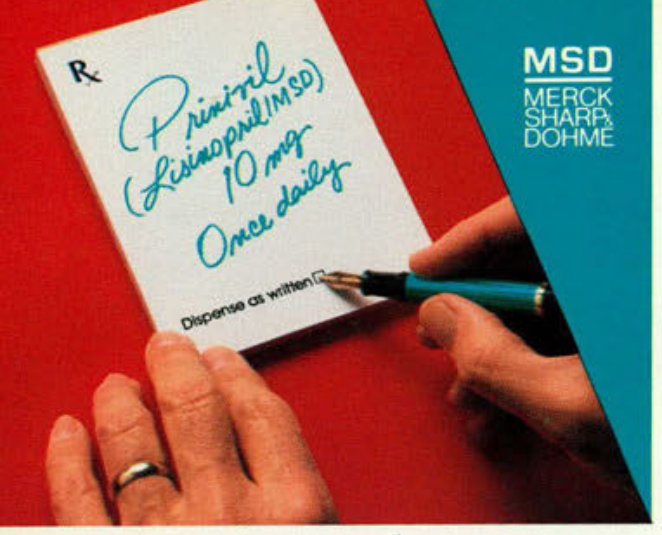

PRINIVIV (LISINOPRIL/MSD)

Available in 5-mg, 10-mg, 20-mg, and 40-mg tablets

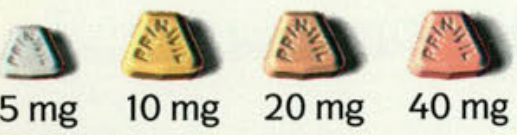

Contraindications:

RINIL (Lisinopril, MSD) is contraindicated in patients who are hypersensitive to this product and in patients with a history of Warnings: glottis, and/or larynx has been reported in patients treated with ACE inhibitors, including PRINIVIL. In such cases, PRINIVIL should

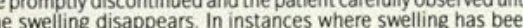
confined to the face and lips, the condition has generally resolved without treatment, although antihistamines have been useful in relieving symptoms. Angioedema associated with laryngea ongue, glottis, or larynx, likely to cause airway obstruction, ppropriate therapy, e.g., subcutaneous epinephrine solution $1: 1000(0.3 \mathrm{~mL}$ to $0.5 \mathrm{~mL})$, should be promptly administered (see ADVERSE REACTIONS) cated hypertensive patients but is a possible consequence of the use of PRINIVIL in salt/volume-depleted persons, such as those CAUTIONS. Drug Interactions and ADVERSE REACTIONS). In patients with severe congestive heart failure, with or without observed and may be associated with oliguria and/or progressive Because of the potential fall in blood pressure in these patients, therapy should be started under very close medical supervision Such patients should be followed closely for the first 2 weeks of treatment and whenever the dose of PRINIVIL and/or diuretic is neart or cerebrovascular disease in whom an excessive fall in blood pressure could result in a myocardial infarction or cerebrovascular accident.

If hypotension occurs, the patient should be placed in a supine position and, if necessary, receive an intravenous infusion of normal saline. A transient hypotensive response is not a contrainddifficulty once the blood pressure has increased after volume expansion.

Anter ACE inhibitor has been shown to cause agranulocytosis and bone marrow depression. with renal impairment, especially if they also have a collagen vaswith renal impairment, especially if they also. Available data from clinical trials of PRINIVIL are insufficient to show that PRINIVIL does not cause agranulocytosis at similar rates. Periodic monitoring of white blood cell counts in patients with collagen vascular disease and renal disease should Precautions:

Ceneral: Impaired Renal Function: As a consequence of inhibiting the renin-angiotensin-aldosterone system, changes in renal function may be anticipated in susceptible individuals. In patients with severe congestive heart failure whose renal function may depend on the activity of the renin-angiotensin-aldosterone system. treatment with ACE inhibitors, including PRINIVIL, may be associated with oliguria and/or progressive azotemia and rarely with acute renal failure and/or death.

In hypertensive patients with unilateral or bilateral renal artery may occur. Experience with another ACE inhibitor suggests that may occur. Experience with another ACE inhibitor suggests that PRINIVIL and/or diuretic therapy. In such patients renal function should be monitored during the first few weeks of therapy.

Some hypertensive patients with no apparent preexisting renal vascular disease have developed increases in blood urea nitrogen and serum creatinine, usually minor and transient, especially when Pre likity has been given concomitantly with a diuretic. This is more likely to occur in patients with preexisting renal impair-.
ment. Dosage reduction of PRINIVIL and/or discontinuation of the

Evaluation of the hypertensive patient should always Evaluation of the hypertensive patient should always
include assessment of renal function (see DOSACE AND include assessment

Hyperkalemia: In clinical trials, hyperkalemia (serum potassium patients and $4.0 \%$ of patients with congestive heart failure. In most cases, these were isolated values which resolved despite of therapy in approximately $0.1 \%$ of hypertensive patients. Risk of therapy in approximately $0.1 \%$ of hypertensive patients. Risk ciency, diabetes mellitus, and the concomitant use of potassium ciency, diabetes mellitus, and the concomitant use of potassiumsparing diuretics. potassium supplements, and/or potassium containing salt substitutes, which should be used cautiously, if
all, with PRINIVIL $*$ (Lisinopril, MSD) (see Drug Interactions). Surgery/Anesthesia: In patients undergoing major surgery or during anesthesia with agents that produce hypotension, PRINIVIL may block angiotensin II formation secondary to compensatory renin release. If hypotension occurs and is considered to be information for Patients:

Angioedema: Angioedema, including laryngeal edema, may occur, especially following the first dose of PRINIVIL. Patients
should be so advised and told to report immediately any signs or should be so advised and told to report immediately any signs or symptoms suggesting angioedema (swelling of face, extremities, no more drug until they have consulted with the prescribing phys cian. report lighthe aypotension. Patients should be cautioned to report lightheadedness, especially during the first few days of continue the drug until they have consulted with the prescribing physician.

All patients should be cautioned that excessive perspiration and dehydration may lead to an excessive fall in blood pressure because of reduction in fluid volume. Other causes of volume blood pressure; patients should be advised to consult with their physician.

Hyperkalemia: Patients should be told not to use salt substitutes containing potassium without consulting their physician

Neutropenia: Patients should be told to report promptly any indication of infectio

NOTE: As with many other drugs, certain advice to patients being treated with PRINIVIL is warranted. This information is intended to aid in the safe and effective use of this medication
disclosure of all possible adverse or intended effects.

Drug Interactions:

Hypotension: Patients on Diuretic Therapy: Patients on diuretics and especially those in whom diuretic therapy was recently instituted, may occasionally experience an excessive reduction of blood pressure after initiation of therapy with PRINIVIL. The pos-
sibility of hypotensive effects with PRINIVIL can be minimized by either discontinuing the diuretic or increasing the salt intake prio to initiation of treatment with PRINIVIL. If it is necessary to condaily and duretic, initiate therapy with PRINIV 2 at a dose initial dose for at least 2 hours and until blood pressure has stabilized for at least an additional hour (see WARNINCS and DOSAGE AND
ADMINISTRATION). When a diuretic is added to the therapy of a patient receiving PRINIVIL, an additional antihypertensive effect is usually observed. Studies with ACE inhibitors in combination with diuretics indicate that the dose of the ACE inhibitor can be reduced when it is

Indomethacin: In a study in 36 patients with mild to moderate hypertension where the antihypertensive effects of PRINIVIL methacin, the use of indomethacin was associated with a reduced effect, although the difference between the two regimens was not

Other Agents: PRINIVIL has been used concomitantly with nitrates and/or digoxin without evidence of clinically significant interactions occurred when PRINIVIL was used concomitantly the stomach does not alter the bioavailability of PRINIVIL.

Agents Increasing Serum Potassium: PRINIVIL attenuates potaspotassium-sparing diuretics (e.g., spironolactone, triamterene, or amiloride), potassium supplements, or potassium-containing salt substitutes may lead to significant
sium. Therefore, if concomitant use of these agents is indicated because of demonstrated hypokalemia, they should be used with caution and with frequent monitoring of serum potassium

Pregnancy Category C: Lisinopril was not teratogenic in mice
treated on days 6 to 15 of gestation with up to $1,000 \mathrm{mg} / \mathrm{kg} / \mathrm{day}$ $(625$ times the maximum recommended human dose). There was an increase in fetal resorptions at doses down to $100 \mathrm{mg} / \mathrm{kg}$; at
doses of $1,000 \mathrm{mg} / \mathrm{kg}$, this was prevented by saline supplementation. There was no fetotoxicity or teratogenicity in rats treated with up to $300 \mathrm{mg} / \mathrm{kg} /$ day ( 188 times the maximum recomreceiving lisinopril from day 15 of gestation through day 21 postpartum, there was an increased incidence in pup deaths on days 2 to 7 postpartum and a lower average body weight of pups on day weight did not occur with maternal saline supplementation. Lisinopril, at doses up to $1 \mathrm{mg} / \mathrm{kg} /$ day, was not teratogenic when given throughout the organogenic period in saline-supplemented
rabbits. Saline supplementation (physiologic saline in place of tap water) was used to eliminate maternotoxic effects and enable evaluation of the teratogenic potential at the highest possible dosage level. The rabbit has been shown to be extremely sensitive to ACE inhibitors (captopril and enalapril) with maternal and fetodosage levels in man. Fetotoxicity was demonstrated in rabbits by an increased incidence of fetal resorptions at an oral dose of lisinopril at I mg/kg/day and by an increased incidence of incomplete ossification at the lowest dose tested $(0.1 \mathrm{mg} / \mathrm{kg} /$ day). A single intravenous dose of $15 \mathrm{mg} / \mathrm{kg}$ of lisinopril administered to preg-
nant rabbits on gestation days 16,21 , or 26 resulted in $88 \%$ to nant rabbits on $\mathrm{g}$
$100 \%$ fetal death

There are no adequate and well-controlled studies in pregnant women. PRINIVIL should be used during pregnancy only if the potential benefit justifies the potential risk to the fetus.

Nursing Mothers: It is not known whether this drug is secreted in human milk. Because many drugs are secreted in human milk. caution should be exercised when PRINIVIL is given to a nursing mother.
Pediatric Use: Safety and effectiveness in children have not been

\section{Adverse Reactions:}

PRINIVIL has been found to be generally well tolerated in conPRINIVIL has been found to be generally well tolerated inc
trolled clinical trials involving 2.003 patients and subjects.
The most frequent clinical adverse experiences in controlled trials with monotherapy with PRINIVI ( Lisinopril, MSD) were: dizziupper respiratory symptoms $(3.0 \%)$, and cough $(2.9 \%)$, all of which were more frequent than in placebo-treated patients. For the most part adverse experiences were mild and transient in nature. Discontinuation of therapy was required in $6 \%$ of patients. In clinical trials, the overall frequency of adverse experiences mended therapeutic dosage range.

rring in greater than $1 \%$ patients and subjects treated with monotherapy with PRINIVIL in controlled clinical trials were: nausea (2.3\%), hypotension chest pain $(1.3 \%)$, vomiting $(1.3 \%)$, and dyspnea $(1.1 \%)$. For information on adverse experiences occurring in greater than $1 \%$ thatients and subjects treated with PRINIL plus hydrochloro Clinical adverse experiences occurring in $0.3 \%$ to $1 \%$ of patients treated with monotherapy with PRINIVIL in the controlled trials and rarer, serious, possibly drug-related events reported in uncontrolled studies or marketing experience included: Body as a pectoris, cardia, peripheral edema, palpitation. Digestive: Abdomina pain, anorexia, constipation, flatulence. Metabolism: Gout. Mus atric: Depression somnolence, insomnia, stroke. Respirator guria, progressive azotemia, acute renal failure. Other: Blurred vision, pruritus, urinary tract infection, vasculitis of the legs. Angioedema: Angioedema has been reported in patients receiving PRINIVIL (0.1\%). Angioedema associated with laryngeal tongue glottis, and/or laryn x occurs, treatment with PRINIVII should be discontinued and appropriate therapy instituted immediately (see WARNINCS). Hypotension: In hypertensive patients,
hypotension occurred in $1.2 \%$ and syncope occurred in $0.1 \%$ of patients. Hypotension or syncope was a cause for discontinuation
of therapy in $0.5 \%$ of hypertensive patients (see WARNINCS). In patients with congestive heart failure, hypotension occurred in $5 \%$ and syncope occurred in $1 \%$ of patients. These adverse experiences were cause for discontinu
patients.

Clinical Laboratory Test Findings:

Serum Electrolytes: Hyperkalemia (see PRECAUTIONS)

Creatinine, Blood Urea Nitrogen: Minor increases in blood urea nitrogen and serum creatinine, reversible upon discontinuation of hypertension treated with PRINIVIL alone. Increases were more common in patients receiving concomitant diuretics and in patients with renal artery stenosis (see PRECAUTIONS). Reverswere observed in approximately $9.1 \%$ of heart failure on concomitant diuretic therapy. Frequently, these abnormalities resolved when the dosage of the diuretic was

Hemoglobin and Hematocrit: Small decreases in hemoglobin and hematocrit (mean decreases of approximately $0.4 \mathrm{~g} \%$ and $1.3 \mathrm{vol}$ $\%$, respectively) occurred frequently in patients treated with some other cause of anemia. In clinical trials, less than $0.1 \%$ of patients discontinued therapy due to anemia.

Other (Causal Relationship Unknown): Rarely, elevations of liver

Overall, $2.0 \%$ of patients discontinued therapy due to laboratory . Dosage and Administration

uncomplicated essential hyperension not on diuretic therapy, the recommended initial dose is $10 \mathrm{mg}$ once a day. Dosage should be adjusted according to biood priministered in a single daily dose. The antihypertensive effect may diminish toward the end of the dosing interval regardless of the administered dose, but most commoniy with a dose of $10 \mathrm{mg}$ daily. This can be evaluated by measuring blood pressure just maintained for 24 hours. If it is not, an increase in dose should be considered. Doses up to $80 \mathrm{mg}$ have been used but do not appear to give a greater effect. If blood pressure is not controlled with L a done, a low dose of a diuretic may be added. ive effect. After the addition of a diuretic, it may be possible to reduce the dose of PRINIVIL

Diuretic-Ireated Patients: In hypertensive patients who are cur-

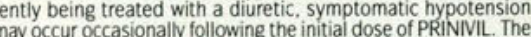
diuretic should be discontinued, if possible, for 2 to 3 days before beginning therapy with PRINIVIL to reduce the likelinood of hypoadjusted according to blood pressure response. If the patient's blood pressure is not controlled with PRIN

If the diuretic cannot be discontinued, an initial dose of $5 \mathrm{mg}$ should be used under medical supervision for at least 2 hours and (see WARNINCS and PRECAUTIONS. Drug Interactions).

Concomitant administration of PRINIVIL with potassium supplemay lead to increases of serum potassium (see PRECAUTIONS) Use in Elderly: In general, blood pressure response and adverse experiences were similar in younger and older patients given simithat maximum blood levels and area under the plasma concentration time curve (AUC) are doubled in older patients so

Dosage Adjustment in Renal Impairment: The usual dose of PRINIVIL ( $(10 \mathrm{mg}$ ) is recommended for patients with a creatinine $3 \mathrm{mg} / \mathrm{dL}$ ). For patients with creatinine clearance $\geq 10 \mathrm{~mL} / \mathrm{min}$ and $3 \mathrm{mg} / \mathrm{dL}$ ). For patients with creatinine clearance $\geq 10 \mathrm{~mL}$ min and
$\leq 30 \mathrm{~mL} / \mathrm{min}$ (serum creatinine $\geq 3 \mathrm{mg} / \mathrm{dL}$ ). the first dose is $5 \mathrm{mg}$ once daily. For patients with creatinine clearance $<10 \mathrm{~mL}$ min usually on hemodialysis), the recommended initial dose is is controlled or to a maximum of $40 \mathrm{mg}$ daily.

For more detailed information, consult your MSD Representative or see Prescribing Information. Merck Sharp \& Dohme. Division of
Merck \& CO., INC., West Point. PA 19486 . 


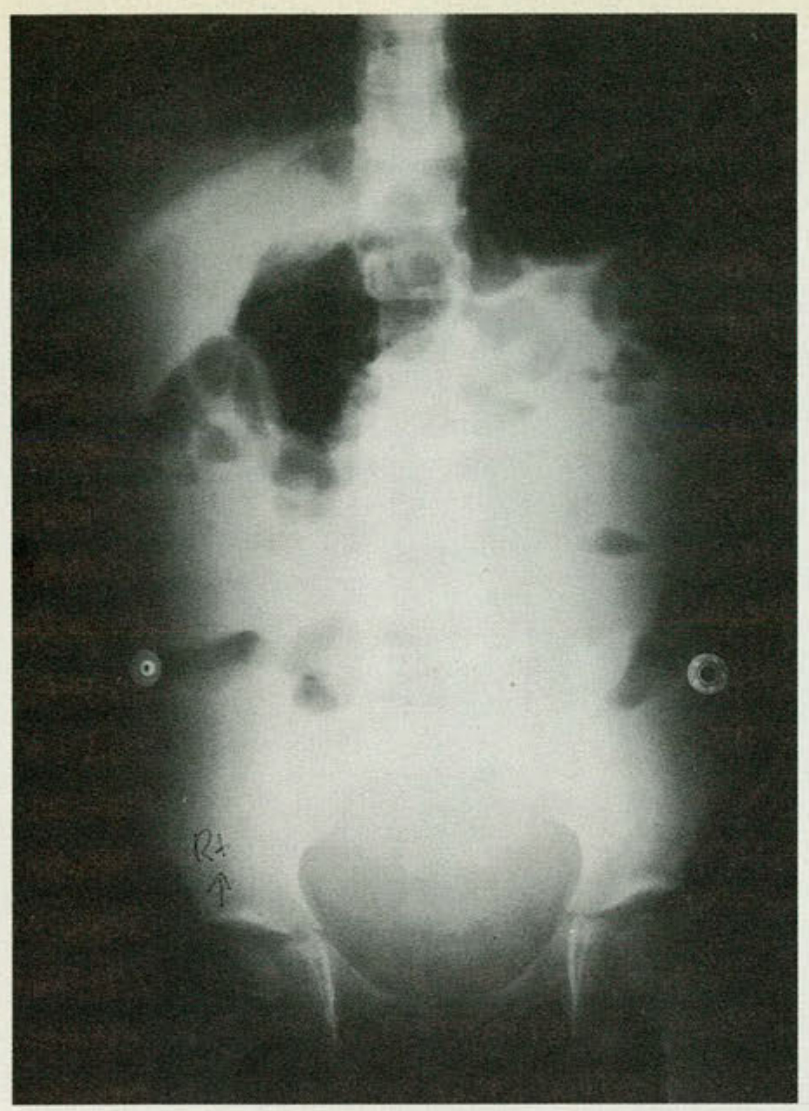

Fig 3. Anteroposterior view showing gas and fluid-filled loops and basic obliteration of organs.

Treatment consists of complete enucleation of the cyst without disruption of surrounding nonpathologic structures. ${ }^{2}$ If the cyst is located close to the abdominal wall, or if obstruction or strangulation of bowel occurs, small-bowel resection may be necessary.

\section{Summary}

Although mesenteric cysts are uncommon, they may be seen by the general practitioner. Multiple episodes of partial small-bowel obstruction or asymptomatic abdominal masses should alert the physician to include mesenteric cysts in the differential

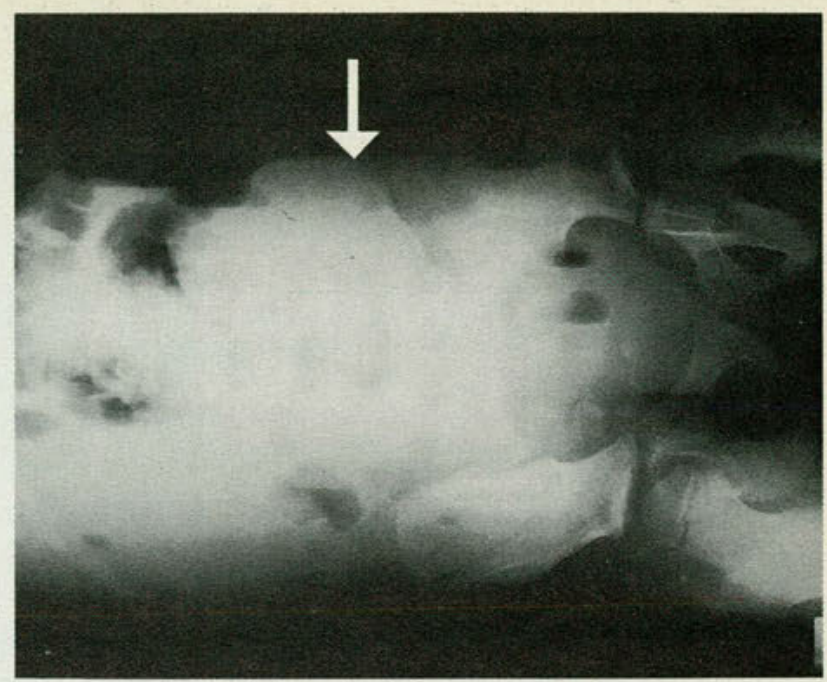

Fig 4. Lateral decubitus view showing the basic outline of the cyst.

diagnosis. Ultrasonography and computed tomography appear to be the most accurate and noninvasive mode of evaluation. As in this case, surgical exploration appears to be the only definitive diagnostic, as well as therapeutic, modality. Postoperative recovery generally is free of sequelae.

1. Rauch RF: Retroperitoneal lymphangioma. Arch Surg 1959;78:45-50. 2. Hardin WJ, Hardy JD: Mesenteric cysts. J Surg 1970;119:640-645. 3. Gallifer RB, Pous JG, Juskiewenski S, et al: Intraabdominal cystic lymphangiomas in childhood. Prog Pediatr Surg 1978;11:131-136.

4. Kurtz RJ, Heimann TM, Beck AR, et al: Mesenteric and retroperitioneal cysts. Ann Surg 1986;203:109-112.

5. Burnett WE, Rosemond GP, Bucher RM: Mesenteric cysts. Arch Surg 1950;60:699-705.

6. Warfield JO Jr: A study of mesenteric cysts, with a report of two recent cases. Ann Surg 1932;96:329-339.

7. Vanek VW, Phillips AK: Retroperitoneal mesenteric and omental cysts Arch Surg 1984;119:838-842.

From the Department of General and Family Practice, Texas College of Osteopathic Medicine, Fort Worth.

Reprint requests to Dr Peterson, TCOM, 3516 Camp Bowie, Fort Worth, TX 76107. 
Foundation (NOF) has the resources you need to help you reshape the future in the prevention and treatment of osteoporosis.

But we need you, too. We need your support to help fund the urgently needed research and educational effort that can conquer a crippling disorder affecting 24 million Americans and resulting in 1.3 million bone fractures annually, including 500,000 vertebral fractures.

For more information about NOF, please mail the coupon at the bottom of this ad. We'll send you materials that describe our goals and achievements; a listing of available literature (including our widely-acclaimed Physicians Resource Man$u a l$ ), and information about our tax-deductible membership fees.

THE ONLY NATIONAL VOLUNTARY HEALTH ORGANIZATION DEDICATED TO OSTEOPOROSIS.
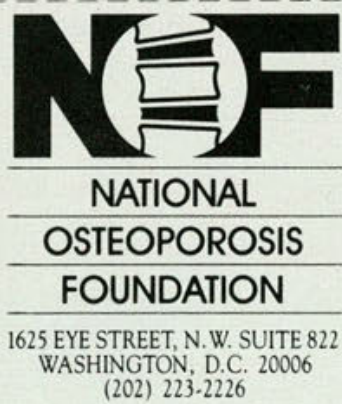

Please send me the NOF information package. 